\title{
Dynamic freezing and defect suppression in the tilted one-dimensional Bose-Hubbard model
}

\author{
U. Divakaran ${ }^{1}$ and K. Sengupta ${ }^{2}$ \\ ${ }^{1}$ Department of Physics, Indian Institute of Technology, Kanpur, India. \\ ${ }^{2}$ Theoretical Physics Department, Indian Association for the Cultivation of Science, Jadavpur, Kolkata-700032, India.
}

\begin{abstract}
We study the dynamics of tilted one-dimensional Bose-Hubbard model for two distinct protocols using numerical diagonalization for finite sized system $(N \leq 18)$. The first protocol involves periodic variation of the effective electric field $E$ seen by the bosons which takes the system twice (per drive cycle) through the intermediate quantum critical point. We show that such a drive leads to nonmonotonic variations of the excitation density $D$ and the wavefunction overlap $F$ at the end of a drive cycle as a function of the drive frequency $\omega_{1}$, relate this effect to a generalized version of Stückelberg interference phenomenon, and identify special frequencies for which $D$ and $1-F$ approach zero leading to near-perfect dynamic freezing phenomenon. The second protocol involves a ramp of both the electric field $E$ (with a rate $\omega_{1}$ ) and the boson hopping parameter $J$ (with a rate $\left.\omega_{2}\right)$ to the quantum critical point. We find that both $D$ and the residual energy $Q$ decrease with increasing $\omega_{2}$; our results thus demonstrate a method of achieving near-adiabatic protocol in an experimentally realizable quantum critical system. We suggest experiments to test our theory.
\end{abstract}

PACS numbers:

\section{INTRODUCTION}

Ultracold atom systems, in the presence of optical lattices, have proved to be successful emulators of several model Hamiltonians such as the Ising and the BoseHubbard models $\stackrel{1}{*}$. These systems offer unprecedented tunability of the parameters of the Hamiltonians they emulate. Consequently, they serve as perfect test bed for studying the low-temperature properties and possible quantum phase transitions of the emulated models 2 . Furthermore, ultracold atom systems provide a near-perfect isolation of its constituents from the environment; this feature, along with real-time tunability of the laser intensity used to create the optical lattice, makes them ideal systems for studying non-equilibrium dynamics of a closed quantum system near its critical point ${ }^{3}$. Several such studies, both experimental and theoretical, have already been undertaken for a system of ultracold bosons in an optical lattice emulating the Bose-Hubbard model near a Mott-insulator (MI)- superfluid (SF) quantum critical point $2,4-\underline{7}$.

More recently, following the theoretical prediction of Ref. 8, there has been experimental realizations of a translational symmetry broken density wave ground state of one-dimensional (1D) ultracold bosons in the presence of an effective electric field ${ }^{9}$. The atoms in the trap are neutral; thus such an electric field can be generated either by shifting the center of the confining trap ${ }^{2}$ or by applying a spatially varying Zeeman magnetic field ${ }^{9}$. It is well-known that the low-energy properties of such a system of bosons can be described by an effective dipole model with a Hamiltonian ${ }^{8}$

$$
H_{d}=-J \sqrt{n_{0}\left(n_{0}+1\right)} \sum_{\ell}\left(d_{\ell}^{\dagger}+d_{\ell}\right)+(U-E) \sum_{\ell} n_{\ell},(1)
$$

where $d_{\ell}=b_{i}^{\dagger} b_{j}$ is the dipole annihilation operator living on the link $\ell$ between sites $i$ and $j$. Such a dipole con- stitutes a bound state of a boson and a hole on adjacent lattice sites $i$ and $j$. Here $b_{i}$ is the boson annihilation operator on site $i, U$ is the on-site repulsion of the bosons, $n_{0}$ is the number of bosons in the MI phase at each lattice site, $J$ is their hopping amplitude, $n_{\ell}=d_{\ell}^{\dagger} d_{\ell}$ is the dipole number operators residing on the link $\ell$, and $E$ is the effective electric field. The dipole operators satisfy additional constraints of having a maximum of single dipole per link $\left(n_{\ell} \leq 1\right)$ and having at most one dipole on two adjacent links $\left(n_{\ell} n_{\ell \pm 1}=0\right)$. These dipole states are resonantly coupled to the parent Mott state when $U \sim E$. It has been shown that such a system undergoes a phase transition from a dipole vacuum state for $U \gg E$ to the one with maximum number of dipoles $(=N / 2$, where $N$ denotes the chain length in units of lattice spacing) when $E \gg U$. This phase transition belongs to the Ising universality class as reported in Ref. 8 and the critical point $E_{c}$ is given by $E_{c}=U+1.31 J \sqrt{n_{0}\left(n_{0}+1\right)}$. It turns out that such a system of bosons also has a description in terms of Ising spins and constitutes the realization of an effective Ising model with both longitudinal $(\sim(U-E))$ and transverse $(\sim J)$ magnetic fields $\stackrel{8.9}{.}$. In the spin language, the dipole vacuum and the maximal dipoles states are termed as paramagnetic (PM) and Ising antiferromagnetic (AFM) respectively ${ }^{\underline{9}}$. Such studies have recently been extended to include the effect of higher dimensions $\stackrel{10}{ }$, disorder $\stackrel{11}{ }$, and for weakly coupled bosons $\frac{12}{2}$.

In the last few years, several studies have been performed to understand different aspects of nonequilibrium dynamics of closed quantum systems. Most of the initial studies in this direction focussed either on sudden quenches $\frac{13-15}{-15}$ or on linear or non-linear ramp protocols taking the systems through quantum critical points $16-22$. The former class of studies were mostly concerned with the evolution and long-time behavior of a closed quantum system following a quench while the lat- 
ter group have demonstrated the possibility of realization of Kibble-Zurek scaling 23,24 and its variants in the context of isolated quantum systems. More recently, several studies have focussed on periodic dynamics of closed quantum systems; such a dynamics inevitably involve multiple passage of a quantum system through the intermediate quantum critical point which leads to novel interference phenomenon 25,26 . Moreover, such protocols lead to the realization of dynamic freezing of the state of the system during a periodic drive $\underline{27,28}$ and may lead to novel steady states 29 . However, most of these studies have not been applied to experimentally realizable non-integrable systems. Further, it has also been recently shown that for a generic quantum Hamiltonian, a two-parameter drive protocol, which constitutes a time dependent ramp of two of the Hamiltonian parameters, may lead to suppression of defects during the passage of a system through a quantum critical point $\underline{30}$. However, such a protocol has never been applied to a specific experimentally relevant and/or non-integrable model.

In this work, we study the non-equilibrium dynamics of the dipole Hamiltonian $H_{d}$ for two separate (periodic and two-rate) protocols using numerical diagonalization for finite sized system $(N \leq 18)$. The former protocol involves periodic variation of the effective electric field $E$ seen by the bosons with a drive frequency $\omega_{1}$ while the latter involves linear ramp of $E$ the and the boson hopping $J$ with rates $\omega_{1}$ and $\omega_{2}$ respectively. We note at the outset that the quench and the ramp dynamics of this model has been studied in Refs. 13 and 31 . The former study has predicted that the long-time average of the dipole order parameter will have a maximal value when the final value of the electric field after the ramp, starting from the dipole vacuum phase, matches with $U$. The latter studies showed that these system could prove as experimental test bed for realization of Kibble-Zurek law for finite size systems. However, the behavior of this model when driven by periodic or two-rate protocols has never been studied. In this work, we aim to fill up this gap.

The main results of our work are as follows. First, we show that a periodic variation of $E$ with a rate $\omega_{1}$, which takes the system twice through the intermediate critical point for each drive period starting from the dipole vacuum phase, leads to non-monotonic variation of the excitation (defect) density $1-F$ (where $F$ is the overlap between the state of the system and the instantaneous ground state at the end of the drive), and the dipole excitation density $D$, measured after an integer number of drive cycles, as a function of $\omega_{1}$. Second, we identify specific frequencies for which $(1-F), D \sim 0$ after a complete drive cycle leading to near-perfect dynamic freezing phenomenon 27,28 . We also show that both these phenomena occurs due to the quantum interference effect originating from multiple passage of the system through the quantum critical point and constitute an example of generalized form of Stückelberg interference phenomenon 26 . Third, for the two-rate protocol, we demonstrate that
$D$ and the residual energy $Q$ exhibit power law dependence on both $\omega_{1}$ and $\omega_{2}$ over a range of drive frequency; they increase (decrease) with increasing $\omega_{1}\left(\omega_{2}\right)$. We identify the corresponding exponents and compare them with the prediction of Kibble-Zurek theory for finite sized system 31 . Finally, we chart out the range of drive frequencies where one expects to experimentally observe such behavior for experimentally relevant finite sized system. We point out that owing to the decrease of $D$ and $Q$ with $\omega_{2}$, such a two-rate protocol may be near-adiabatic and thus might be useful for quantum state preparation in an experimentally realizable system upon its passage through a quantum critical point. We note that both the non-monotonic behavior of $D$ and $F$ for the periodic protocol and the suppression of $D$ and $Q$ with increasing $\omega_{2}$ provide examples of phenomena that have no analog in standard quench and ramp protocols; thus we expect our results to provide additional relevant information for possible future experiments in this system.

The plan of the rest of the paper is as follows. In Sec. III we chart out the details of the protocols studied and the method of our analysis. This is followed by Sec. III. where we present the main results for periodic and two rate dynamics. Finally we conclude with a discussion of our main results and their experimental relevance in Sec. IV.

\section{ANALYSIS OF THE TILTED BOSE HUBBARD MODEL}

The boson Hubbard model, in the presence of an effective electric field $E$, which essentially tilts the lattice along one direction, can be written as

$$
\begin{aligned}
H= & -J \sum_{\langle i j\rangle}\left(b_{i}^{\dagger} b_{j}+b_{j}^{\dagger} b_{i}\right)+\frac{U}{2} \sum_{j} n_{j}\left(n_{j}-1\right) \\
& -E \sum_{j} j n_{j} .
\end{aligned}
$$

Here $n_{j}=b_{j}^{\dagger} b_{j}$ denotes the number of bosons at site $j$, and $U$ is the on-site repulsive interaction potential. As shown in Ref. 8, starting from the parent Mott state with $n_{0}$ bosons per site, the low energy behavior of the tilted Bose Hubbard model, for $U, E \gg|U-E|, J$, can be described by the effective dipole Hamiltonian $H_{d}$ given by Eq. 1. In what follows we shall analyze the dynamics of the dipoles in the presence of time-dependent hopping strength $J(t)$ and electric field $E(t)$ such that $U, E(t) \gg$ $|U-E(t)|, J(t)$ at all times so that $H_{d}$ can be reliably used to describe the dynamics of the dipoles. We shall use numerical exact diagonalization technique for obtaining eigenvalues and eigenfunctions of $H_{d}$; this limits the size of the system to $N \leq 18$. We note such system sizes are similar to what has been experimentally achieved in Ref. 9; thus our results are expected to be of direct relevance to possible future experiments on these systems. 
We first consider the periodic protocol for which

$$
E(t)=U-E_{0} \cos \left(\omega_{1} t\right),
$$

where $E_{0}$ is chosen such that the system starts in a dipole vacuum state at $t=0$. Note that with this choice of the protocol, the instantaneous energy of dipole formation, $U-E(t)$, vanishes twice at $\omega_{1} t=\pi / 2,3 \pi / 2$ during each drive cycle. Also, the system crosses critical point when $E(t)=E_{c}$; this also occurs twice at $t=t_{1,2}$ for each drive cycle, where

$$
\omega_{1} t_{1}=\cos ^{-1}\left(-\mu_{0} J / E_{0}\right), \quad t_{2}=2 \pi / \omega_{1}-t_{1},
$$

where $\mu_{0}=1.3 \sqrt{n_{0}\left(n_{0}+1\right)}$. The Schrödinger equation for the many-body wavefunction $|\psi\rangle$ in the presence of a periodic $E(t)$ is given by

$$
i \hbar \partial_{t}|\psi(t)\rangle=H_{d}[E(t)]|\psi(t)\rangle
$$

To solve Eq. 5, we expand $|\psi(t)\rangle=\sum_{n} c_{\alpha}(t)|\alpha\rangle$, where $|\alpha\rangle$ denotes the instantaneous eigenstates of $H_{d}$ for $E=$ $E(t=0)$. These eigenstates satisfy $H_{d}(t=0)|\alpha\rangle=$ $\epsilon_{\alpha}|\alpha\rangle$, where $\epsilon_{\alpha}$ denotes the instantaneous eigenenergies at $t=0$. Here the coefficients $c_{\alpha}(t)$ represent the overlap of the state $|\psi(t)\rangle$ with $|\alpha\rangle$. Eq. 5 can now be reexpressed as coupled set of differential equations governing the time evolution of $c_{\alpha}(t)$. These equations are given by

$$
\begin{aligned}
\left(i \hbar \partial_{t}-\epsilon_{\alpha}\right) c_{\alpha} & =E_{0}\left[1-\cos \left(\omega_{1} t\right)\right] \sum_{\beta} \Lambda_{\alpha \beta}^{(1)} c_{\beta}(t), \\
\Lambda_{\alpha \beta}^{(1)} & =\left\langle\alpha\left|\sum_{\ell} n_{\ell}\right| \beta\right\rangle
\end{aligned}
$$

with the initial condition $c_{\alpha}(0)=c_{0 \alpha}$. The coefficient $c_{0 \alpha}$, and the eigenenergies $\epsilon_{\alpha}$ are obtained by exact diagonalization of $H_{d}(t=0)$. A numerical solution of these equations yields the state of the system $|\psi(t)\rangle$ at any time $t$ during the drive.

Having obtained $|\psi(t)\rangle$, one can use it to compute expectation values of several relevant quantities. In the present work, we shall mainly concentrate on the wavefunction overlap $F$, the dipole excitation density $D$, and the residual energy $Q$ of the system. In terms of the overlap coefficients $c_{\alpha}(t)$, one can obtain these quantities as

$$
\begin{aligned}
D(t) & =\left|n_{d}(t)-\left\langle\psi_{G}(t)\left|\sum_{\ell} n_{\ell}\right| \psi_{G}(t)\right\rangle / N\right| \\
& =\left|n_{d}(t)-n_{d}^{G}(t)\right| \\
n_{d}(t) & =\frac{1}{N}\left\langle\psi(t)\left|\sum_{\ell} n_{\ell}\right| \psi(t)\right\rangle=\frac{1}{N} \sum_{\alpha \beta} c_{\alpha}^{*}(t) c_{\beta}(t) \Lambda_{\alpha \beta}^{(1)} \\
F(t) & =\left|\left\langle\psi(t) \mid \psi_{G}(t)\right\rangle\right|^{2}, \\
Q(t) & =\langle\psi(t)|H(t)| \psi(t)\rangle-\left\langle\psi_{G}(t)|H(t)| \psi_{G}(t)\right\rangle \\
& =\sum_{\alpha \beta} c_{\alpha}^{*}(t) c_{\beta}(t)\langle\alpha|H(t)| \beta\rangle-\left\langle\psi_{G}(t)|H(t)| \psi_{G}(t)\right\rangle,
\end{aligned}
$$

where $\left|\psi_{G}(t)\right\rangle$ is the instantaneous ground state of the system at time $t, n_{d}$ is the dipole density at time $t, n_{d}^{G}$ is the dipole density corresponding to the instantaneous ground state at $t$, and we have set the lattice spacing to unity. We note that the expressions of $n_{d}, D, F$ and $Q$ obtained in Eq. 8 assume a particularly simple form when evaluated at the end of an integer $(p)$ number of drive cycles, i.e., at $t=t_{f}=2 \pi p / \omega_{1}$. This simplicity arises from the fact that $H\left(t_{f}\right)=H(0)$ in these cases and leads to

$$
\begin{aligned}
n_{d}\left(t_{f}\right) & =\frac{1}{N} \sum_{\alpha \beta} c_{\alpha}^{*}\left(t_{f}\right) c_{\beta}\left(t_{f}\right) \Lambda_{\alpha \beta}^{(1)}, \quad F\left(t_{f}\right)=\left|c_{0}\left(t_{f}\right)\right|^{2} \\
D\left(t_{f}\right) & =\left|n_{d}\left(t_{f}\right)-\Lambda_{00}^{(1)} / N\right| \\
Q(t) & =\sum_{\alpha \neq 0}\left(\epsilon_{\alpha}-\epsilon_{0}\right)\left|c_{\alpha}\left(t_{f}\right)\right|^{2}
\end{aligned}
$$

where we have denoted the initial ground state of the system by $|\alpha=0\rangle \equiv|0\rangle$.

Finally, we consider the two rate protocol where one varies both the boson hopping amplitude $J$ and the effective electric field $E$ according to the protocol

$$
E(t)=E_{i}+\Delta E \omega_{1} t, \quad J(t)=\epsilon+\Delta J \omega_{2} t
$$

where the initial value of the electric field $E_{i}$ is chosen so that the system is in the paramagnetic phase, and $\epsilon / \Delta J$ is chosen to be a small number. The ramp starts at $t=0$ and continues till $t=t_{c}$ when the system reaches the critical point: $E\left(t_{c}\right)=U+\mu_{0} J\left(t_{c}\right)$. This yields

$$
\begin{aligned}
t_{c} & =\left(U-E_{i}-\mu_{0} \epsilon\right) / \omega_{0}, \\
\omega_{0} & =\left(\omega_{1} \Delta E-\mu_{0} \omega_{2} \Delta J\right) .
\end{aligned}
$$

We note that for $\omega_{2}>\omega_{2}^{c}=\omega_{1} \Delta E /\left(\mu_{0} \Delta J\right), \omega_{0}<0$ leading to $t_{c}<0$ which indicates that for fast enough $\omega_{2}$ the system is not going to reach the critical point. In this work, we shall restrict ourselves to $\omega_{2} \leq \omega_{2}^{c}$.

To obtain the solution of Eq. 5 we follow a procedure similar to the case of the periodic single parameter drive and expand the wavefunction $|\psi(t)\rangle=\sum_{\alpha} c_{\alpha}^{\prime}(t)|\alpha\rangle$, where $|\alpha\rangle$ denotes the eigenstates of $H(t=0)$ with $E=E_{i}$ and $J=\epsilon$ satisfying $H|\alpha\rangle=\epsilon_{\alpha}^{\prime}|\alpha\rangle$. Eq. 5 then leads to the coupled equations of motion for $c_{\alpha}^{\prime}(t)$

$$
\begin{aligned}
\left(i \hbar \partial_{t}-\epsilon_{\alpha}^{\prime}\right) c_{\alpha}^{\prime}(t) & =-t \sum_{\beta}\left(\Delta E \omega_{1} \Lambda_{\alpha \beta}^{(1)}+\Delta J \omega_{2} \Lambda_{\alpha \beta}^{(2)}\right) c_{\beta}^{\prime}(t) \\
\Lambda_{\alpha \beta}^{(2)} & =\left\langle\alpha\left|\sum_{\ell}\left(d_{\ell}+d_{\ell}^{\dagger}\right)\right| \beta\right\rangle
\end{aligned}
$$

with the initial condition $c_{\alpha}^{\prime}(t=0)=c_{0 \alpha}^{\prime}$. These equations can be solved numerically and $c_{0 \alpha}^{\prime}$ and $\epsilon_{\alpha}^{\prime}$ can be obtained by exact diagonalization of $H_{d}(t=0)$. This procedure leads to $|\psi(t)\rangle$ and hence, via Eq. 8 to $n_{d}, Q$, $D$ and $F$.

\section{RESULTS}

In this section, we discuss the results obtained by numerical analysis of Eq. 6 and 11. In Sec. IIIA, we discuss our results involving compuattion of $D$ and $F$ for 
the periodic protocol (Eq. 3). This is followed by Sec. IIIB, where we numerically compute $D$ and $Q$ for tworate protocol (Eq. 9).

\section{A. Periodic Protocol}

In the presence of the periodic drive (Eq. 31), the instantaneous ground state of the tilted Bose Hubbard model changes from zero dipole (PM) to the maximum dipole (AFM) state and back, passing twice through the intermediate quantum critical point for each drive cycle. For this protocol, $\omega_{1} \rightarrow 0$ corresponds to the adiabatic limit where system remains close to the instantaneous ground state at all times.

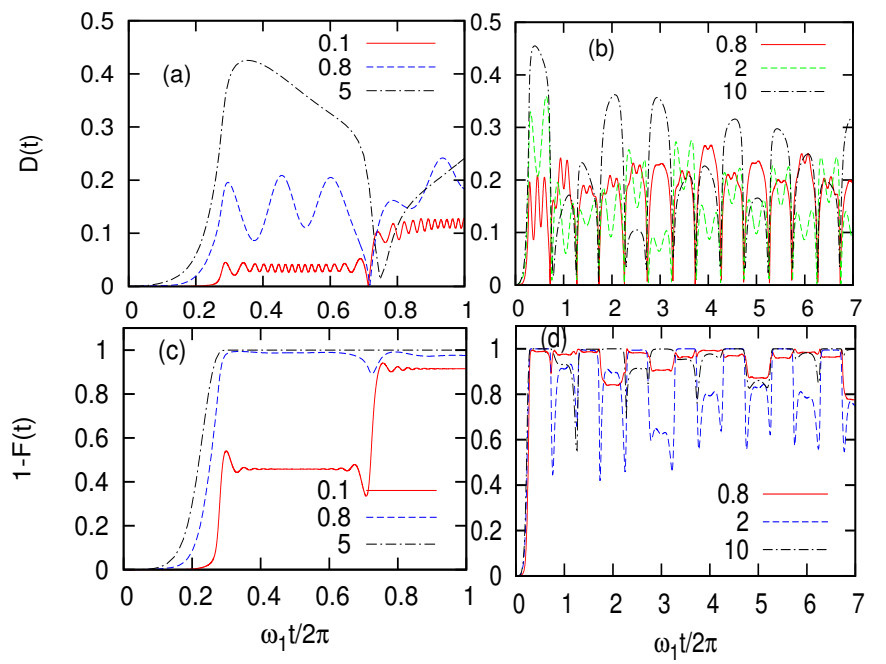

FIG. 1: (a) and (b): Plot of variation of the dipole excitation density $D(t)$ as a function of $\omega_{1} t$ for different scaled frequency $\omega_{1} /\left(E_{c}-U\right)$ (shown in the figure) up to one (a) and seven (b) drive periods. (c) and (d): Plots of the defect density $1-F(t)$ as a function of $\omega_{1} t$ for same parameters as (a) and (b) respectively.

We first present our results obtained through numerical simulations as described in the previous section for $\left(U-E_{0}\right) / J=30$. In Fig. 1(a), we plot the time evolution of dipole excitation density $D$ as a function of time $t$ for one complete cycle and for several scaled frequencies $\omega_{1} /\left(E_{c}-U\right)$. As can be seen from the figure, and as theoretically expected, fewer defects are generated during the drive for smaller frequencies. For a fixed frequency, $D=\left|n_{d}(t)-n_{d}^{G}(t)\right|$ starts increasing when the critical region is crossed for the first time (around $t=t_{1}$ ). This happens since for small frequencies, the system enters the impulse region around the critical point where the state of the system starts to deviate from the instantaneous ground state (which, for $E \leq E_{c}$ has $n_{d}^{G}(t) \simeq 0$ ) leading to increase in $D$. As we continue the evolution within the AFM phase, the system stays in an excited state with $n_{d}(t)<n_{d}^{G}(t)$ for $t_{1}<t<t_{2}$, with higher $D(t)$ for larger frequencies. After crossing the critical point for the second time and reaching the PM phase, the reverse condition is true with $n_{d}(t)>n_{d}^{G}(t)(\sim 0)$. This leads to a dip in $D(t)$ when $n_{d}(t)=n_{d}^{G}(t)$. We find numerically that in the periodic case, the dip in $D(t)$ occurs at $E=E^{*} \simeq E_{c}$, especially for small frequencies as shown in Fig. 2. We note however, that the fact $D \simeq 0$ does not mean that the state of the system is identical to the instantaneous ground state at this point and does not constitute an example of dynamical freezing. This can be seen from a plot of $1-F$ as a function of $t$; we find that $1-F$ does not approach zero concomitantly with $D$. The corresponding behavior of $1-F$ as a function of $t$ is shown in Fig. 1(c).

Next, we study the behavior of $D$ and $1-F$ after a complete cycle of drive as a function of $\omega_{1}$. The results are shown in Fig. 3. We find that both $D$ and $1-F$, after a complete drive cycle, display non-monotonic oscillatory behavior as a function of $\omega_{1}$. Also, as can be seen in Fig $3(a)$, there are certain special frequencies at which $1-F(T)$ and $D(T)$, where $T=2 \pi / \omega_{1}$, concomitantly approach zero signalling near perfect revival of the wavefunction. This phenomenon is termed as dynamics induced freezing in Ref. 28. To explore how close one approaches near perfect freezing in the present system, we plot $\ln D \equiv \ln D(T)$ and $\ln (1-F) \equiv \ln [1-F(T)]$ as a function of $\omega_{1}$ in Fig. 3. As can be seen in Fig 3(a), $\ln (1-F)$ and $\ln D$ can be as low as -10 for small $\omega_{1}$. In Fig. 3(b), one finds that the freezing is effective for $\omega_{1} /\left|U-E_{c}\right| \ll 1$; for larger frequencies, the freezing phenomenon disappears and $1-F$ and $D$ decreases monotonically with $\omega_{1}$. In particular, in the large frequency regime, both $D,(1-F) \sim \omega_{1}^{-2}$.

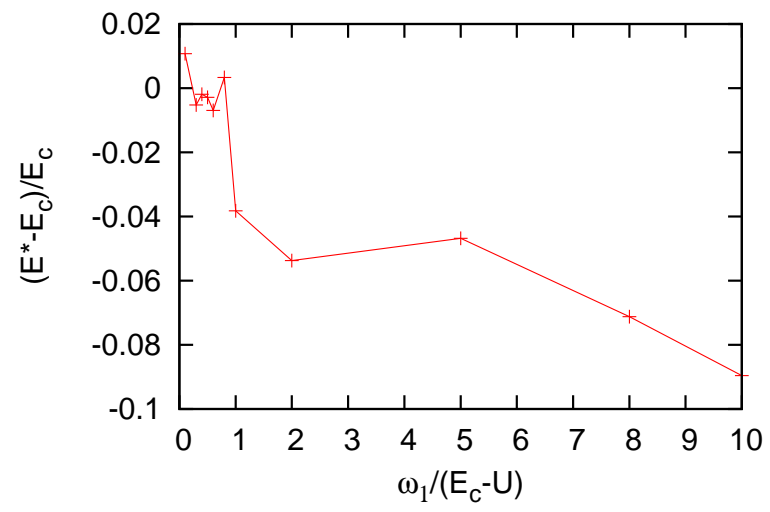

FIG. 2: Plot of the position of the dip in $D,\left(E^{*}-E_{c}\right) / E_{c}$, as a function of scaled frequency $\omega_{1} /\left(E_{c}-U\right)$. The dip is closer to the critical point for smaller frequencies.

The non-monotonic dependence of $D$ and $(1-F)$ as a function of the drive frequency $\omega_{1}$ is a reminiscent of the analogous behavior of the probability of excitation for two level systems subjected to periodic drives. This behavior originates from the interference effect between probability amplitude of the two-level system wavefunction at the ground and the excited state on second pas- 


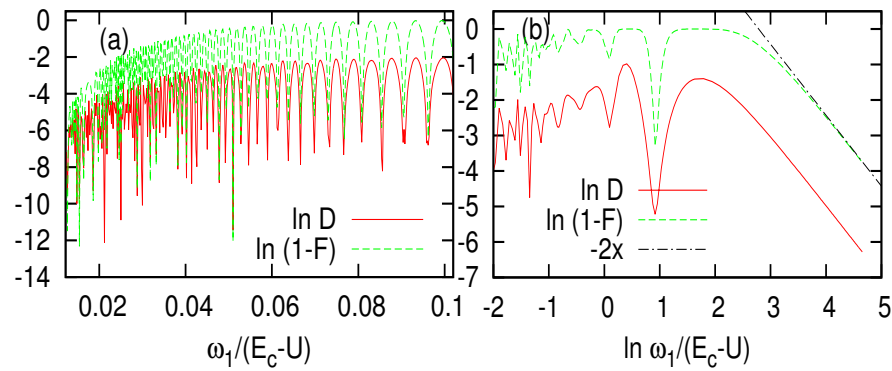

FIG. 3: Plot of $\ln (D)$ and $\ln (1-F)$ after one complete cycle as a function of $\omega_{1}$. (a) corresponds to small frequency region where for certain frequencies, $F$ is close to unity (or $1-F \sim 0$ ) which signals near perfect freezing of the wavefunction after one complete cycle. As the frequency increases, the freezing phenomena becomes less effective and ultimately disappears for $\ln \left[\omega_{1} /\left(E_{c}-U\right)\right]>2$ as shown in (b).

sage through the avoided level crossing during the periodic drive. Such an interference phenomenon is known as Stückelberg interference ${ }^{26}$. However, the system of dipoles at hand is a many-body system with several energy levels whose number increases with system size. Thus, it is a priori unclear whether the oscillatory behavior of $D$ and $F$ observed here can also be explained in terms of such interference phenomenon between a few states. To understand this further, we therefore analyze the wavefunction overlap $\left|c_{\alpha}^{2}(t)\right|=|\langle\psi(t) \mid \alpha\rangle|^{2}$, where $|\psi(t)\rangle$ is the state of the system after time $t$ and $|\alpha\rangle$ are the eigenstates of $H(t=0)$. A plot of $\left|c_{\alpha}(t)\right|^{2}$ for some selected $|\alpha\rangle$ satisfying $\left|c_{\alpha}(t)\right|^{2} \geq 10^{-5}$ at any time $t$ during the evolution is shown in Fig. 4 for two representative frequencies $\omega_{1} /\left|U-E_{c}\right|=0.051$ and 0.074 and for system size $N=14$. The first of these frequencies correspond to a dip in $D$ and $(1-F)$ while the second to their peak. We find that in both cases the system starts in the state $|\alpha\rangle=|0\rangle$ so that $\left|c_{0}(t)\right|^{2} \simeq 1$ until the first passage through the critical point at $t=t_{1}$. During the first passage, a few other $\left|c_{\alpha}(t)\right|^{2}$ develops non-zero value as shown in Fig. 4 . This is then followed by a regime $t_{1} \leq t \leq t_{2}$, where the system is close to the AFM ground state having maximum probability $\left|c_{\alpha}\right|^{2}$ for $\alpha=842$ which corresponds to the AFM ground state. As the system approaches $t=t_{2}$ where it completes its second passage through the critical point, we find that there is again a transfer of weight between several states. These features are common for both frequencies. However, the crucial difference between the two cases lies in the fact that for $t>t_{2}|\psi(T)\rangle$ has near perfect overlap with $|\alpha=0\rangle$ (dipole vacuum ground state) when $\omega_{1} /\left|U-E_{c}\right|=0.051$ with $\left\langle n_{d}\right\rangle=0$; in contrast, for $\omega_{1} /\left|U-E_{c}\right|=0.074$, it has a substantial overlap with $|\alpha=15\rangle$ which corresponds to $\left\langle n_{d}\right\rangle \simeq 2.1$. The final state also has a non-zero overlap with $|\alpha=14\rangle$ having $\left\langle n_{d}\right\rangle=1$.1. This difference originates from the quantum interference between the several states which gains a finite probability amplitude during the second passage of the system through the critical point. We have checked
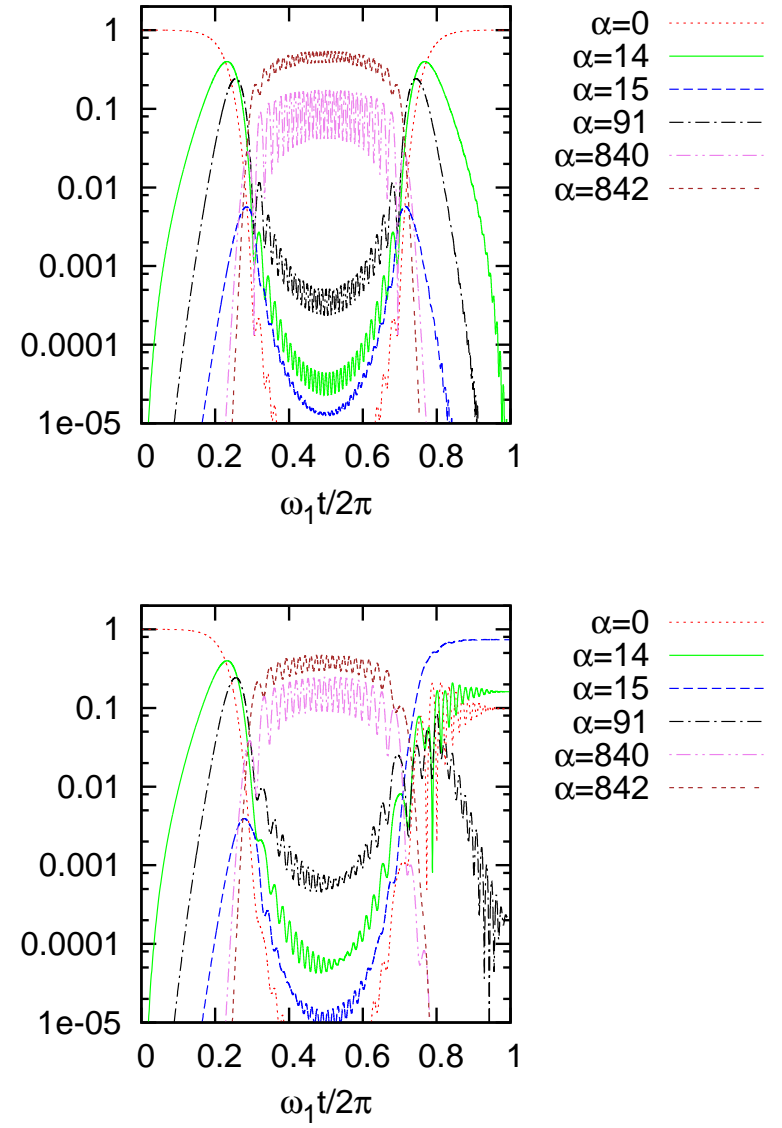

FIG. 4: Plot of the wavefunction overlap coefficients $\left|c_{\alpha}(t)\right|^{2}$ for selected set of $\alpha$ satisfying the condition $\left|c_{\alpha}(t)\right|^{2} \geq 1 E-5$ as a function of $\omega_{1} t$ during the drive cycle. The top figure corresponds to $\left.\omega_{1} /\left(E_{c}-U\right)\right)=0.051$ corresponding to a minima of $D$ while that at the bottom has $\omega_{1} /\left(E_{C}-U\right)=0.074$ corresponding to a maxima of $D$.

that in between these two frequencies which corresponds to a maxima or a minima of $D$ and $1-F,|\psi(T)\rangle$ always remain a superposition of three states. In other words, it is possible to write

$$
\begin{aligned}
|\psi(T)\rangle \simeq & c_{0}\left(\omega_{1}\right)|\alpha=0\rangle+c_{14}\left(\omega_{1}\right)|\alpha=14\rangle \\
& +c_{15}\left(\omega_{1}\right)|\alpha=15\rangle
\end{aligned}
$$

so that

$$
\begin{aligned}
& F(T) \simeq\left|c_{0}\left(\omega_{1}\right)\right|^{2} \\
& D(T) \simeq\left(n_{14}\left|c_{14}\left(\omega_{1}\right)\right|^{2}+n_{15}\left|c_{15}\left(\omega_{1}\right)\right|^{2}\right) / N,
\end{aligned}
$$

where in the last line we have assumed that the ground state at $t=T$ is a zero dipole state, and $n_{14}$ and $n_{15}$ are the number of dipoles in the $|\alpha=14\rangle$ and $|\alpha=15\rangle$, respectively. We compare $D$ and $F$ obtained from Eq. 13 with the numerical calculations performed in Fig. 5 and observe a very good agreement between the two.

Thus, we find that the dynamics of the many-body system at the end of a drive cycle can be described 

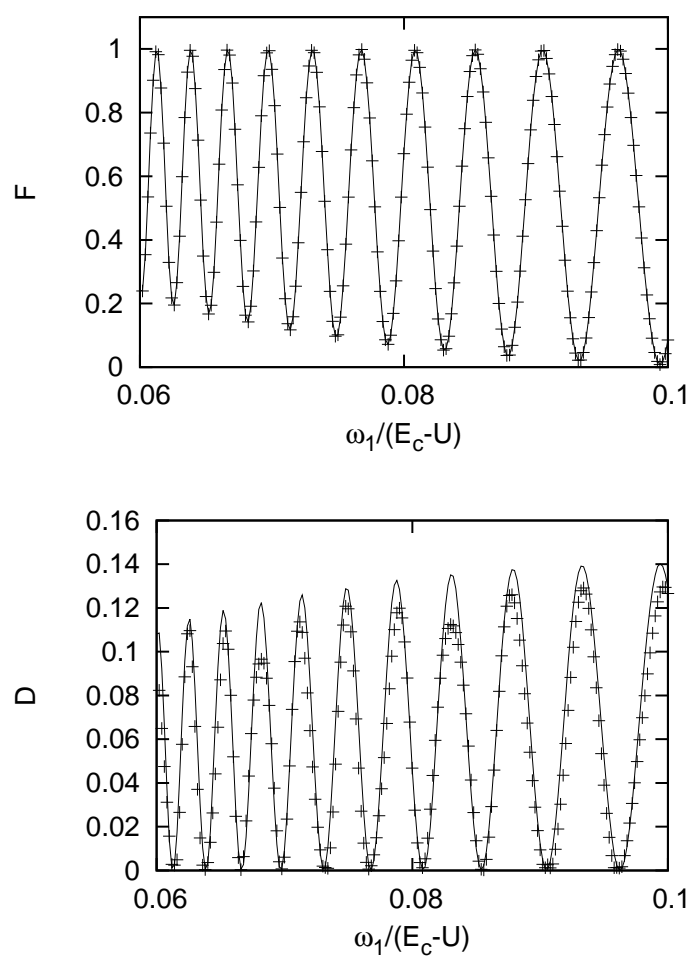

FIG. 5: Plots of the wavefunction overlap $F$ (top) and the dipole excitation density $D$ (bottom) as a function of $\omega_{1} /\left(E_{c}-\right.$ $U)$ showing near perfect match between analytical (Eq. 13) and exact numerical calculations. The points correspond to numerical results and the lines to Eq. 13 .

by an effective three-level model since the wavefunction after the drive is controlled by the coefficients $c_{0}\left(\omega_{1}\right)$, $c_{14}\left(\omega_{1}\right)$ and $c_{15}\left(\omega_{1}\right)$. Numerically, as shown in Fig. [6. for a range of $\omega_{1}$, these coefficients display oscillatory behavior as a function of $\omega_{1}$ which results in the oscillatory behavior of $D$ and $1-F$. Also, we note that the phenomenon of dynamic freezing occurs for $\omega_{1}=\omega^{*}$ for which $\left|c_{0}\left(\omega^{*}\right)\right|^{2} \simeq 1$. Hence, we observe a phenomenon which is a modified version of the Stückelberg interference for the following reasons. First, similar to the Stückelberg interference phenomenon, the probability of the system to return to the ground state can be described in terms of a few states (one needs three states here compared to two states in the usual descriptions of Stückelberg interference). Second, the occupation probabilities of these three states display an oscillatory behavior leading to oscillations of $D$ and $F$. Third, the weight transfer between these states originates from quantum interference between several many-body states upon second passage through a quantum critical point. However, in contrast to the usual two-level systems where such a phenomenon is first predicted ${ }^{26}$, the weight transfer between the dipole states at the critical points involve several many-body states; thus the dependence of $c_{0}, c_{14}$ and $c_{15}$ on $\omega_{1}$ is determined by interference between multiple many-body states which, in contrast to the original
Stückelberg problem, does not easily admit an analytical description. However, our analysis at least establishes the fact that the near-perfect dynamic freezing observed in this system originates from quantum interference between many-body states during multiple (two) passages of the system through the PM-AFM quantum critical point. We note in passing that we have checked that qualitatively similar phenomenon occurs for other values of $\omega_{1}$ corresponding to maxima or minima of $D$ and $1-F$ and for other system sizes $N=12,16$, and 18 .

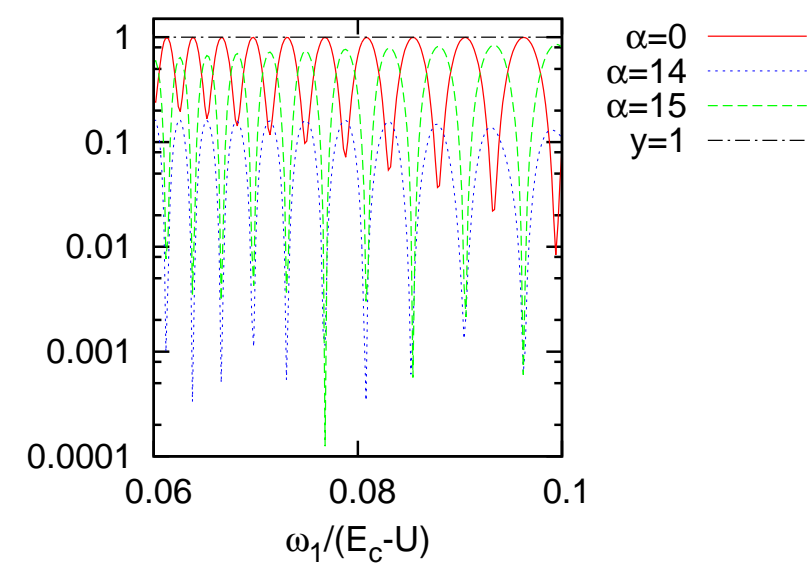

FIG. 6: Plot of the variation of $\left|c_{\alpha}\right|^{2}$ at $t=T$ as a function of $\omega_{1}$ for $\alpha=0,14$ and 15 .

\section{B. Two-rate Protocol}

In this section, we study the dynamics of the system in the presence of the two-rate protocol given by Eq. 9 . We note that recently such a protocol has been shown to provide a mechanism for defect suppression in Ref. 30 for integrable models. In the case of integrable models, the two parameters of the Hamiltonian were varied with rates $\omega_{1}$ and $\omega_{2}$ so that the system crosses the quantum critical point at some time $t_{c}$. It was shown that for these models, one of the time dependent parameters controlled the proximity of the system to the quantum critical point whereas the other controlled the dispersion of the quasiparticle at the critical point with rate $\omega_{2}$. It was also shown that the defect density $D$ and the residual energy $Q$ for a $d$-dimensional system obey novel universal power-law behavior given by

$$
D \sim \omega_{1}^{\frac{(2 z \nu+1) d}{z(1+z \nu)}} \omega_{2}^{-d / z}, Q \sim \omega_{1}^{\frac{(2 z \nu+1)(d+z)}{z(1+z \nu)}} \omega_{2}^{-(d+z) / z}(14)
$$

where $\nu$ and $z$ are the critical exponents related to correlation length and correlation time, respectively. Note that both $D$ and $Q$ decrease with $\omega_{2}$.

We now apply the two-rate protocol to the tilted Bose Hubbard model. The drive protocol is given by Eq. 9 . We start from an initial PM ground state corresponding 
to $\left(U-E_{i}\right) / \Delta J=100$ at $t=0$, with $\Delta E / \Delta J \simeq 200$, and $\epsilon / \Delta J=0.001$. Both $E(t)$ and $J(t)$ are varied with two different velocities till the critical point at time $t_{c}$ which is given by Eq. 10. The crucial difference of the present case which constitutes an example of a non-integrable model is that the microscopic parameters $J$ and $E$ differ from those of the effective theory controlling the low-energy dynamics.

To identify the parameters of the effective theory which controls the proximity to the critical point and the velocity or dispersion of the quasiparticle in this model, we define the instantaneous quasiparticle gap $\Delta(t)=$ $U-E(t)+\mu_{0} J(t)$ in the PM phase. We note that $\Delta\left(t_{c}\right)=0$. Expanding $\Delta(t)$ around $t=t_{c}$, one finds $|\Delta(t)| \sim\left|\omega_{0}\left(t-t_{c}\right)\right|$, where $\omega_{0}$ is given by Eq. 10. Thus, we find that it is $\omega_{0}$ (and not $\omega_{1}$ or $\omega_{2}$ ) which controls the proximity to the critical point. Next, we identify the term which controls the dispersion. As shown in Ref. 8, the instantaneous velocity of the dipoles in the PM phase is given by $J(t)^{2} /|U-E(t)|$. Thus, the velocity of quasiparticles around the critical point can be estimated to be $v \sim J\left(t_{c}\right) \propto\left(\omega_{2} / \omega_{0}\right)$. We have verified our estimates for the gap and the velocity numerically by studying the gap at the critical point for a finite system. Identifying $J\left(t_{c}\right)$ as the quasiparticle velocity near the quantum critical point, and following the arguments in Ref. 30, it is then straightforward to obtain

$$
D \sim \omega_{0}^{3 / 2} \omega_{2}^{-1} \text { and } Q \sim \omega_{0}^{3} \omega_{2}^{-2}
$$

In terms of the experimental frequencies $\omega_{1}$ and $\omega_{2}$, one thus expects

$$
\begin{aligned}
& D \simeq\left(\omega_{1}-\nu_{0} \omega_{2}\right)^{3 / 2} \omega_{2}^{-1} \\
& Q \simeq\left(\omega_{1}-\nu_{0} \omega_{2}\right)^{3} \omega_{2}^{-2}
\end{aligned}
$$

where $\nu_{0} / \Delta J=\mu_{0} / \Delta E$. For $\nu_{0} \ll \omega_{1} / \omega_{2}, \omega_{0} \simeq \omega_{1}$ and one recovers the scaling relations of Eq. 14 .

We now present numerical results obtained by solving Eq. 11. To check the predictions outlined in Eq. 16. we first set $\omega_{2}=\omega_{1}^{r}$. In this case, $D \sim \omega_{1}^{3 / 2}(1-$ $\left.\nu_{0} \omega_{1}^{r-1}\right)^{3 / 2} \omega_{1}^{-r}$. Once again, for $\nu_{0} \ll \omega_{1}^{1-r}$, Eq. 16 predicts a crossover in variation of $D$ as a function of $\omega_{1}$. For $r<3 / 2, D$ should increase with $\omega_{1}$ whereas it is expected to decrease with $\omega_{1}$ for $r>3 / 2$. On the other hand, at $r=3 / 2, D$ should be a universal number which is independent of $\omega_{1}$. A plot of $D$ vs $\omega_{1}$, shown in Fig. 7 for $n_{0}=1$, confirms this behavior for different $r$. We note that the decrease of $D$ with $\omega_{1}$ for $r>3 / 2$ shows that it is possible to realize a near-adiabatic protocol by tuning microscopic parameters $J$ and $E$ in a non-integrable quantum many-body system.

Next, we study the dynamics of the system keeping $\omega_{1}$ and $\omega_{2}$ independent so that the predicted behavior in Eq. 16 with each of them can be verified. First, we fix $\omega_{2}$ and plot the variation of $\ln D$ and $\ln (Q / N)$ as a function of $\ln \left[\omega_{1} /\left(U-E_{i}\right)\right]$ for different system sizes $18 \geq N \geq 14$ and for several representative values of $\omega_{2}$ in Figs. 8 and 9. These plots show the expected increase of $D$ and $Q$ as

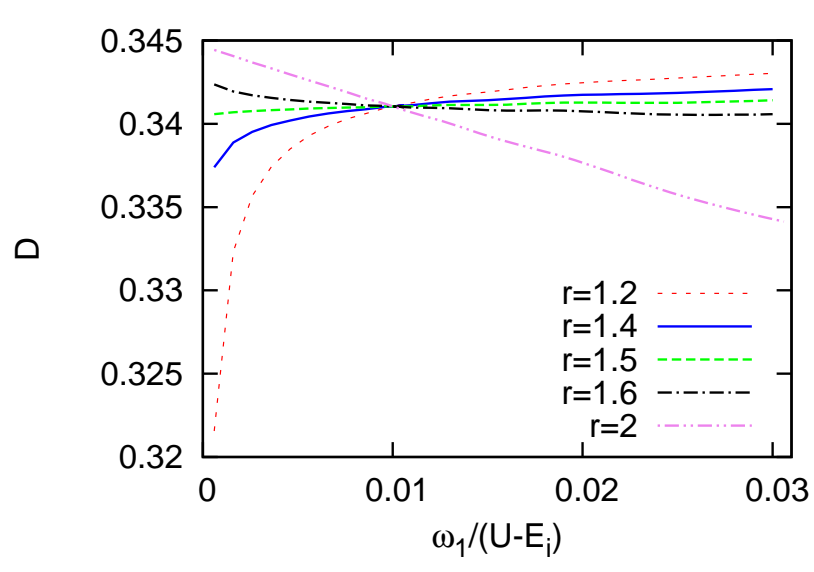

FIG. 7: Plot of the variation of the dipole excitation density $D$ with $\omega_{1}$ for different $r$ with fixed $\omega_{2}=\omega_{1}^{r}$. The plot shows a clear crossover in behavior of $D$ from increasing to decreasing function of $\omega_{1}$ as $r$ passes through $3 / 2$. Here we have set $\nu_{0}=0.01$.

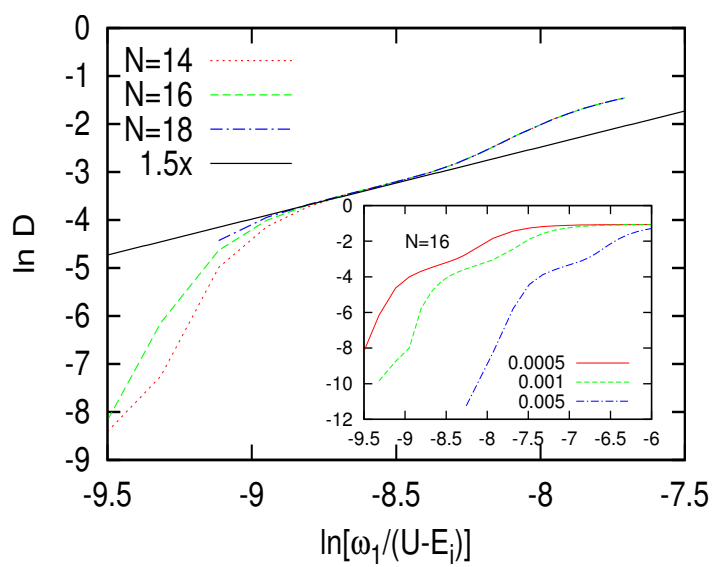

FIG. 8: Plot of $\ln (D)$ with $\omega_{1}$ for different system sizes and fixed $\omega_{2} /\left(U-E_{i}\right)=0.0005$. The ramp of $E$ and $J$ starts from $U-E_{i}=100$ to $U-E_{f}$ where $E_{f}$ is the value of the effective electric field at the critical point as discussed in the text. The scaling behavior of $D$ predicted in Eq. 16 is over a finite frequency range $-8.4 \geq \ln \left(\omega_{1} /\left(U-E_{i}\right)\right) \geq-9.0$; see text for details. Inset: Plot of $\ln (D)$ as a function of $\omega_{1}$ for $\omega_{2} /\left(U-E_{i}\right)=0.0005$ (red solid line), 0.001 (green dashed line) and 0.005 (blue dash-dotted line) with system size set to $N=16$. All other parameters are same as in Fig. 7

a function of $\omega_{1}$. We note from these plots that both $D$ and $Q$ follow the expected Kibble-Zurek scaling behavior for a finite intermediate range of $\omega_{1}$ whose value depend on $\omega_{2}$. For example, for $\omega_{2}=0.05$ in Fig. 8, we find this range to be $-8.4 \geq \ln \left(\omega_{1} /\left(U-E_{i}\right)\right) \geq-9.0$. However for lower $\omega_{1}$, both $D$ and $Q$ deviates from this scaling behavior. This can be understood as a finite-size effect. As shown in Ref. 31, the scaling relation for $D$ and $Q$ are modified by appropriate scaling functions due to finitesized effect which leads to Landau-Zener type behavior $\left(D \sim \omega_{1}^{-2}\right)$ for $\omega_{1} L \ll 1$. Physically, this can be under- 


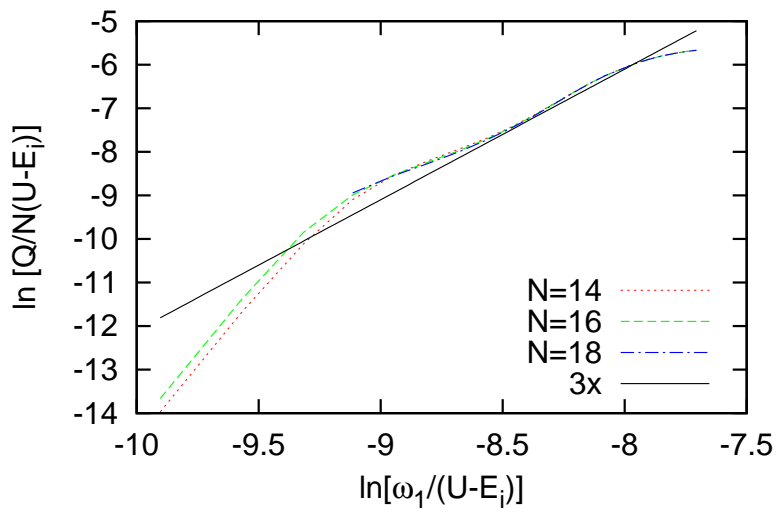

FIG. 9: Plot of $\ln \left[Q /\left(N\left(U-E_{i}\right)\right)\right]$ with $\omega_{1}$ for different system sizes and fixed $\omega_{2} /\left(U-E_{i}\right)=0.0005$ demonstrating the agreement between the theoretically predicted $Q \sim \omega_{1}^{3}$ and the numerical simulations over a range of $\omega_{1}$ (see text for details). All other parameters are same as in Fig. 7.

stood as the presence of gap in the energy spectrum at the critical point due to finite-size effect. This gap, which originates from the presence of a lower momentum cutoff $\sim 1 / L$, leads to an avoided level crossing which leads to Landau-Zener type dynamics for $\omega L^{2} \ll 1$ $\omega_{1}, D$ reaches a plateau as a function of $\omega_{1}$ signalling the setting in of the sudden quench regime where the response of the system becomes independent of $\omega_{1}$.

Finally, we present the numerical results for the dependence of $D$ on $\omega_{2}$ as shown in Fig. 10. We again find agreement between the theoretically expected behavior $D \sim \omega_{2}^{-1}$ (note that we have set $\nu_{0} \ll 1$ ) for a range of drive frequency $-5.5 \leq \ln \left(\omega_{2} /\left(U-E_{i}\right)\right) \leq-4.7$. As also found for $\omega_{1}$ dependence of $D$, scaling behavior does not hold for smaller frequencies $\ln \left(\omega_{2} /\left(U-E_{i}\right)\right) \leq-5.5$ suggesting setting in of finite size effects. For larger frequencies $\ln \left(\omega_{2} /\left(U-E_{i}\right)\right) \geq-4.7, D$ registers a sharper drop than $\omega_{2}^{-1}$ suggesting the end of scaling regime. We note that for the entire range, $D$ is a monotonically decreasing function of $\omega_{2}$ which indicates larger excitation suppression with increasing $\omega_{2}$. We have checked that $F$ and $Q$ show qualitatively similar behavior as a function of $\omega_{2}$.

\section{DISCUSSION}

In this work, we have studied the behavior of bosons in a tilted one-dimensional optical lattice in the presence of both periodic and two-rate drives using exact diagonalization and for finite size systems $N \leq 18$. For the periodic drive protocol which takes the system twice through the intermediate critical point separating the paramagnetic (dipole vacuum) and the ferromagnetic (maximal dipole) states, we have demonstrated the presence of nonmonotonic dependence of the dipole excitation density $D$ and the defect density $1-F$ (where $F$ is the wave-

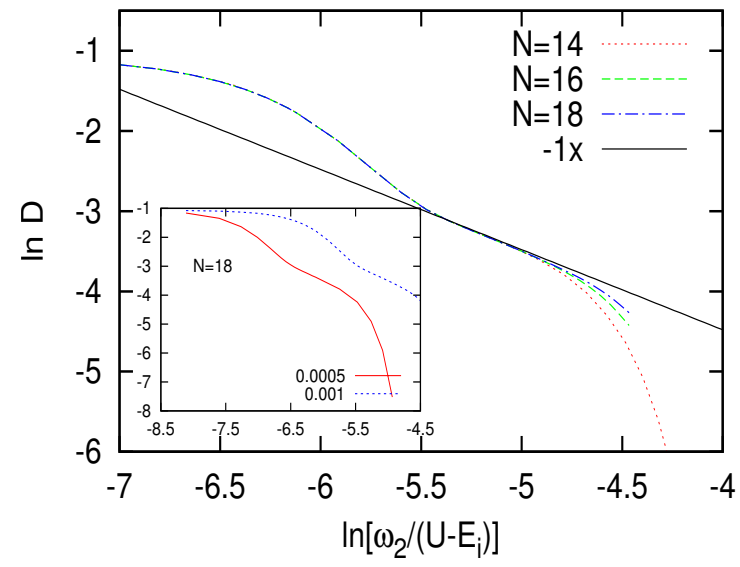

FIG. 10: Plot of $\ln (D)$ as a function of rate $\omega_{2}$ for different system sizes and fixed $\omega_{1} /\left(U-E_{i}\right)=0.001$ showing decrease of $D$ with $\omega_{2}$. The theoretically predicted slope obtained from the scaling theory is also shown for comparison. The figure also demonstrates the role of finite size effects; the $N=18$ data follows the power law till larger value of $\omega_{2}$. Inset: Plot of $\ln (D)$ as a function of $\omega_{2}$ for fixed $N=18$ and $\omega_{1} /\left(U-E_{i}\right)=$ 0.0005 (red solid line) and 0.001 (blue dotted line). All other parameters are same as in Fig. 7.

function overlap between the final state after the drive and the initial ground state) as measured at the end of a complete drive cycle. We have shown that such a behavior originates from quantum interference between the wavefunctions of different states of the boson Hilbert space and constitutes a many-body generalization of the Stückelberg interference phenomenon for two-level systems. Our work also identifies special frequencies where such an interference phenomenon leads to near zero values of $D$ and $1-F$; at these frequencies the system exhibits a near-perfect dynamic freezing $\ln (1-F) \sim-8$ in the sense that the system wavefunction, at the end of a drive period, has a near perfect overlap with the starting ground state wavefunction. We note that such an interference phenomenon has no analog in ramp ${ }^{31}$ or quench ${ }^{13}$ dynamics of the models studied earlier.

For the two-rate protocol, which constitutes a ramp of both the electric field $E$ and the hopping amplitude $J$ of the bosons taking the system from the paramagnetic (dipole vacuum) phase to the critical point, we demonstrate suppression of dipole excitation density $D$ as a function of $\omega_{2}$. We demonstrate that $D$ is a monotonically decreasing function of $\omega_{2}$ and chart out the scaling regime where $D \sim \omega_{2}^{-1}$ for a fixed $\omega_{1}$. We also study the behavior of the system by setting $\omega_{2}=\omega_{1}^{r}$ and demonstrate that the system exhibits a crossover at $r=3 / 2$. For $r<3 / 2, D$ increases with $\omega_{1}$ while it decreases for $r>3 / 2$; at $r=3 / 2 D$ is a constant. We note that our results constitutes an experimentally realizable demonstration for defect suppression on passage of a many-body system through a quantum critical point.

Finally, we discuss possible experiments which can test our theory. In this respect, we note that tilted experi- 
mental lattice systems has already been experimentally studied for $N=12$ in Ref. 9; in particular, the ground state phase diagram of the model has been experimentally verified using direct measurement of on-site parity of occupation of the bosons. More recently, other techniques which allows for direct measurement of boson occupation at a given site has also been reported 32 . Our suggested experiments are build on these and are as follows. First, we suggest measurement of dipole density for the periodically driven titled lattice system where the effective electric field is varied periodically as a function of time. Such an electric field is realized in experiments by using a spatially varying Zeeman field; consequently, its periodic variation can be achieved by making the Zeeman field a periodic function of time. We suggest periodic tuning of the electric field from a value $E_{i}$ which corresponds to the dipole vacuum state (or the $n=1$ Mott phase of the bosons) through the phase transition value $E_{c}$ followed by subsequent measurement of number of sites, $n_{\text {even }}$, with even boson occupation number (which corresponds to the dipole density $D$ ) at the end of a period of the drive. Our theoretical prediction is that $n_{\text {even }}$ shall be a periodic function of the drive frequency $\omega_{0}$. We also predict the existence of specific values of $\omega_{0}$ where the system shall exhibit near-perfect dynamic freezing leading to $n_{\text {even }} \rightarrow 0$. Second, we suggest a linear ramp protocol for variation of the hopping amplitude of these bosons $J$ (with rate $\omega_{2}$ ) and the effective electric field $E$ (with rate $\omega_{1}$ ) which takes the system from the dipole vacuum phase to the critical point. We note that such a protocol can be achieved by simultaneous linear ramp of the applied Zeeman field and the laser intensity controlling the depth of the optical lattice. For this protocol, we predict $n_{\text {even }}$ at the end of the ramp will be a monotonically decreasing function of $\omega_{2}$ for a fixed $\omega_{1}$.

In conclusion, we have studied the response of bosons in a tilted optical lattice in the presence of periodic and two-rate protocols. For the periodic protocol, we have identified special frequencies at which the system exhibits dynamic freezing and have related this phenomenon to a many-body version of Stückelberg interferece effect. For the two-rate protocol, we have identified drive frequency ranges at which the finite-size systems displays scaling behavior as predicted by Kibble-Zureck theory. We have also demonstrated that an increase of $J$ with a rate $\omega_{2}$ leads to decrease of $D$ and $Q$ leading to realization of a near-adiabatic protocol for this system on passage through a quantum critical point. We have also suggested concrete experiments which can test our theory.

\section{Acknowledgments}

UD gratefully acknowledges funding from DSTINSPIRE Faculty fellowship by DST, Govt. of India, and the hospitality of IACS, Kolkata, during her visits.
1 I. Bloch, J. Dalibard, and W. Zwerger, Rev. Mod. Phys. 80, 885 (2008).

2 M Greiner, O Mandel, T Esslinger, TW Hänsch, I Bloch, Nature 415, 39, (2002); C. Orzel, A. K. Tuchman, M. L. Fenselau, M. Yasuda and M. A. Kasevich, Science 291, 2386 (2001).

3 A. Polkovnikov, K. Sengupta, A. Silva and M. Vengalattore, Rev. Mod. Phys. 83, 863 (2011).

4 M.P.A. Fisher, P.B. Weichman, G. Grinstein, and D.S. Fisher, Phys. Rev. B 40, 546 (1989); K. Sheshadri, H. R. Krishnamurthy, R. Pandit and T. V. Ramakrishnan, Europhys. Lett. 22, 257 (1993); J. Freericks and P. Monien, Europhys. Lett 26, 545 (1995).

${ }^{5}$ K. Sengupta and N. Dupuis, Phys. Rev. A 71, 033629 (2005); J. K. Freericks, H. R. Krishnamurthy, Y. Kato, N. Kawashima, and N. Trivedi, Phys. Rev. A 79, 053631 (2009).

${ }^{6}$ C. Trefzger and K. Sengupta, Phys. Rev. Lett 106, 095702 (2011); A. Dutta, R. Sensarma and K. Sengupta, arXiv:1406.0849 (unpublished).

7 W. Bakr, A. Peng, E. Tai, R. Ma, J. Simon, J. Gillen, S. Foelling, L. Pollet, and M. Greiner, Science 329, 547-550 (2010).

8 S. Sachdev, K. Sengupta, and S. M. Girvin, Phys. Rev. B 66, 075128 (2002).

9 J. Simon, W. Bakr, R. Ma, M. E. Tai, P. Preiss and M. Greiner, Nature (London) 472, 307 (2011).

10 S. Pielawa, T. Kitagawa, E. Berg, and S. Sachdev, Phys.
Rev. B 83, 205135 (2011).

11 S. Pielawa, E. Berg, and S. Sachdev, Phys. Rev. B 86, 184435 (2012)

12 C. P. Rubbo, S. R. Manmana, B. M. Peden, M. J. Holland, and A. M. Rey, Phys. Rev. A 84, 033638 (2011)

13 K. Sengupta, S. Powell, and S. Sachdev. Phys. Rev. A 69, 053616 (2004).

14 P. Calabrese and J. Cardy, J. Stat. Mech.: Theory and Experiment06 P06008 (2005).

15 P. Calabrese and J. Cardy. Phys. Rev. Lett 96, 136801 (2006).

16 W. H. Zurek, U Dorner, and P. Zoller, Phys. Rev. Lett. 95, 10570, (2005).

17 A. Polkovnikov, Phys. Rev. B 72, R161201 (2005).

18 D. Sen, K. Sengupta and S. Mondal, Phys. Rev. Lett 101, 016806 (2008).

19 K. Sengupta, D. Sen and S. Mondal, Phys. Rev. Lett. 100, 077204 (2008).

20 A. Dutta, U. Divakaran, D. Sen, B. K. Chakrabarti, T. F. Rosenbaum and G. Aeppli, arXiv:1012.0653 (unpublished).

21 V. Mukherjee, U. Divakaran, A. Dutta and D. Sen, Phys. Rev. B 76, 174303 (2007); U. Divakaran, V. Mukherjee, A. Dutta and D. Sen, J. Stat. Mech., P11001 (2007).

22 A. Polkovnikov, Phys. Rev. Lett. 101, 220402 (2008)

23 T. W. B. Kibble, J. Phys. A 9, 1387 (1976);

${ }^{24}$ W. H. Zurek, Nature (London) 317, 505 (1985).

25 V. Mukherjee and A. Dutta, J. Stat. Mech., P05005 (2009). 
26 S.N. Shevchenko, S. Ashhab and Franco Nori, Phys. Rept. 492, 1 (2010).

27 A. Das, Phys. Rev. B 82, 172402 (2010).

28 S. Mondal, D. Pekker and K. Sengupta, EuroPhys. Lett. 100, 60007 (2012).

29 A. Lazarides, A. Das, and R. Moessner, arXiv:1403.2946 (unpublished).
30 J. D. Sau and K. Sengupta, arXiv:1402.4805 (unpublished).

31 M. Kolodrubetz, D. Pekker, B. K. Clark, and K. Sengupta. Phys. Rev. B 85, 100505 (2012).

32 Y. S. Patil, L. M. Aycock, S. Chakram, and M. Vengalattore, arXiv:1404.5583 (unpublished). 